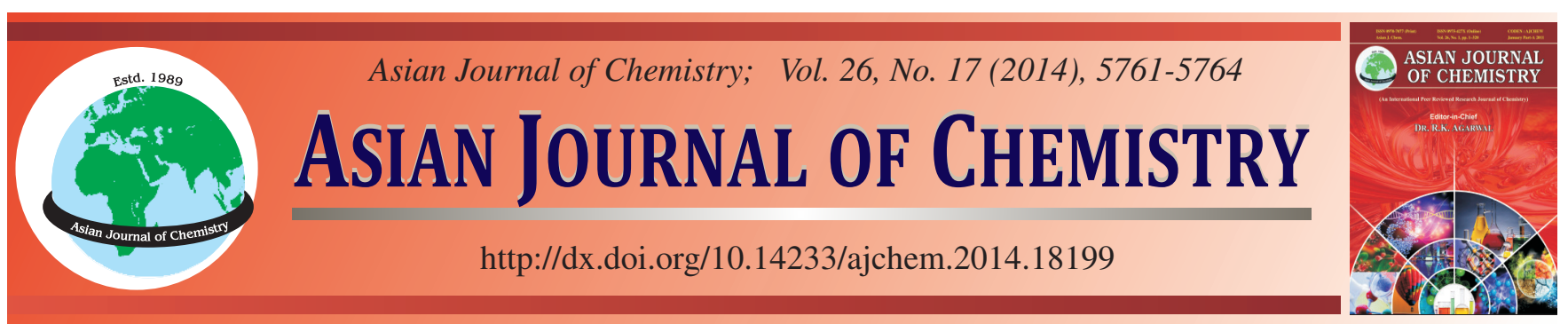

\title{
Magnetic Properties of Carbon-Encapsulated Ni Core-Shell Structure Nanoparticles by DC Arc Plasma $\dagger$
}

\author{
X.J. WU ${ }^{1,2}$, Z.Q. WeI ${ }^{1,2, *}$ X.J. ChEN ${ }^{1}$, X. WANG ${ }^{2}$, W.J. FenG ${ }^{2}$, H. YANG ${ }^{1,2}$ and J.L. JIANG ${ }^{2}$
}

${ }^{1}$ State Key Laboratory of Gansu Advanced New Non-ferrous Metal Materials, Lanzhou University of Technology, Lanzhou 730050, P.R. China ${ }^{2}$ School of Science, Lanzhou University of Technology, Lanzhou 730050, P.R. China

*Corresponding author: Fax: +86 931 2976040; Tel: +86931 2973780; E-mail: zqwei7411@163.com

\begin{abstract}
Carbon encapsulated nickel nanoparticles were prepared via DC arc plasma process. The composition, morphologies, microstructure, particle size and magnetic properties of the particles were characterized via X-ray diffraction (XRD), high-resolution transmission electron microscopy (HRTEM), X-ray energy dispersive spectrometry (XEDS) and vibrating sample magnetometer (VSM). The experiment results show that the carbon encapsulated nickel nanoparticles possesses clear core-shell structure. The core of the particles is face centered cubic structure Ni and the shell is carbon. The particles are scattered homogeneously in spherical or ellipsoidal shapes. The particle size of the nanocapsules ranges from $15-40 \mathrm{~nm}$ with the average value about $30 \mathrm{~nm}$. The particle diameter of the core is about 18 $\mathrm{nm}$ and the thickness of the shells is about $6-8 \mathrm{~nm}$ and the magnetic properties of the products are paramagnetic at room temperature.
\end{abstract}

Keywords: Carbon-encapsulated, Nanoparticles, DC arc plasma, Microstructure, Magnetic properties.

\section{INTRODUCTION}

The metal nanoparticles have attracted significant attention due to their novel physical and chemical properties and it have a broad range of promising applications, such as ultrahigh-density magnetic recording media, environmental protection, biomedicine, catalysis, magnetic toners for xerography, field oriented drug delivery systems and magnetic fluid ${ }^{1-4}$, etc. However, the pure metal nanoparticles are active and unstable, easy to be agglomerated, oxidized in air and dissolved in acid, which limit their potential applications in industry or academic and technological studies. Depositing a protective shell has been recommended as one prospective method to overcome these limitations. Various coating materials have been proposed, e.g., metal oxides, silica, titanium, boron oxide and polymers ${ }^{5-8}$. Carbon seems to be the most desired material for encapsulation, because carbon-encapsulated metal nanoparticles retain their intrinsic nanocrystalline properties and offer a good opportunity to investigate the dimensionally confined systems. In addition, these effectively prevent the agglomeration and oxidation of the core metal nanoparticles due to the high steady encapsulation shell ${ }^{9,10}$. Many methods have been applied to prepare carbon-encapsulated metal magnetic nanoparticles, such as solution phase chemical reduction, instant pyrolysis, arc discharge, microwave heating, radio frequency thermal plasma, explosion, pyrolysis and chemical vapour condensation $^{11-17}$, etc. However, it is well known that most of the reported experimental procedures for the synthesis of nanopowders were still limited in laboratory scale due to some unresolved problems, such as unique reaction conditions, tedious procedures, the complex apparatus, low-yield and high-cost. In addition, byproducts such as carbon nanotubes, nanofibers, nanoparticles and free amorphous carbon were likely to form by using these techniques. Therefore, from a practical viewpoint, it is vital to develop a more efficient and low cost method to fabrication carbon-encapsulated metal nanoparticles. Arc discharge method is a conventional and versatile process, which has been widely used for the synthesis of metal nanoparticles, alloyed, metal-oxide system fullerenes and carbon nanotubes $^{18,19}$.

In this study, DC arc plasma equipment was modified, the electrode device was devised in a vertical state, the graphite crucible was tightly installed in the inner of the water-cooled copper crucible and served as the anode, the cathode was the plasma gun, which was mainly made up of the graphite rod and the water-cooled nozzle, the ionized gases were confined through the nozzle outlet to form the plasma jet. Carbonencapsulated nickel nanoparticles were successfully prepared 
by using modified DC arc plasma technique in an inert atmosphere. In addition, the composition, morphology, microstructure, particle size and magnetic properties of the products were studied.

\section{EXPERIMENTAL}

A schematic diagram of the experimental installation designed to obtain carbon-encapsulated nickel nanoparticles was fully illustrated elsewhere ${ }^{20}$. It mainly includes the stainless steel vacuum chamber, the gas supply device, the DC power supply and the plasma gun with a high frequency ignited device, the vacuum pump, the water-cooled crucible and the water-cooled collection cylinder. Before the experiment, the synthesis chamber was evacuated to a base pressure of $10^{-3} \mathrm{~Pa}$ and diluted twice by Ar, then filled with $\mathrm{Ar}$ to a wanted pressure. The arc in an inert environment was automatically ignited between the graphite electrode and the crucible by the high frequency ignited device, which was maintained by the current source at the preestablished values of the voltage and current. The bulk metal Ni was heated and melted by the high temperature of the plasma, when the kinetic energy of metal atoms exceeded the metal superficial energy, metal atoms detached from the metal surface and evaporated into free atom state. The vaporization of $\mathrm{Ni}$ and gasification of carbon occurred synchronously, a plasma plume was produced with super highdensity $\mathrm{Ni}$ vapour, gasified carbon and argon species. Above the evaporation source was a region of supersaturated vapour, where the free atoms diffused around and collided with each other to decrease the nucleus forming energy. When the metal vapour supersaturated, a new phase was nucleated homogeneously out of the aerosol systems. The droplets were rapidly cooled and combined to form primary particles by an aggregation growth mechanism. The carbon encapsulated $\mathrm{Ni}$ nanoparticles were formed by free diffusion of internal solid state carbon to the surface and deposition of external gas phase carbon on the particles outside. The particles were transported from the nucleation and growth area to the inner walls of the cylinder by the free inert gas convection between the hot evaporation source and the cooled collection cylinder. The dispersed carbon-encapsulated Ni nanoparticles were collected at the inner walls of the water-cooled collection cylinder after a period of stabilization with working gas.

The crystalline structures of the products were characterized by a rotating-target X-ray diffractometer (Japan Rigaku $\mathrm{D} / \mathrm{Max}-2400)$ with graphite monochromatized $\mathrm{CuK}_{\alpha}$ radiation $(\lambda=1.54056 \AA, 40 \mathrm{kV}, 100 \mathrm{~mA})$. The samples were scanned in the angular range from $10-90^{\circ}(2 \theta)$ with scanning rate $0.005^{\circ} \mathrm{s}$ and step size $0.02^{\circ}$. The average crystalline grain size of the metal core was estimated from the half maximum width and the peak position of an XRD line broadened according the Scherrer formula. The morphology and microstructure of the sample were observed through JEM-2010 high-resolution transmission electron microscopy (HRTEM). The chemical composition of the products was analyzed by energy dispersive spectroscopy (XEDS) at an acceleration voltage of $200 \mathrm{keV}$ in TEM. Magnetic hysteresis loops were investigated at room temperature using a vibrating sample magnetometer (VSM, TOEI 5S 15) with an applied field from +15000 Oe to 15000 Oe.

\section{RESULTS AND DISCUSSION}

Fig. 1 shows the representative high resolution electronic microscope microstructure (HRTEM) morphology and microstructure of carbon-encapsulated Ni nanoparticles. It can be seen from Fig. 1(a) that carbon-encapsulated Ni nanoparticles possess spherical or ellipsoidal shapes, with smooth surface, uniform size and good dispersion. Most nanocapsules possess distinct shell-core structure with isolated cores. The diameter of the nanocapsules ranges from 15 to $40 \mathrm{~nm}$, with an average diameter about $30 \mathrm{~nm}$. The diameter of the core ranges from several nanometers to $30 \mathrm{~nm}$, the mean particle size of the core is about $18 \mathrm{~nm}$ and the thickness of the outer carbon shell is about 6-8 $\mathrm{nm}$. According to the HRTEM micrograph, except for carbon-encapsulated Ni nanoparticles, no carbon nanotubes or nanowires, empty carbon cages or other unwanted byproducts are observed. The HRTEM micrograph proved that DC arc plasma technique is an efficient method to obtain carbon-encapsulated Ni nanoparticles, which has successfully overcome the problem of agglomeration and oxidation of the core metal nanoparticles.
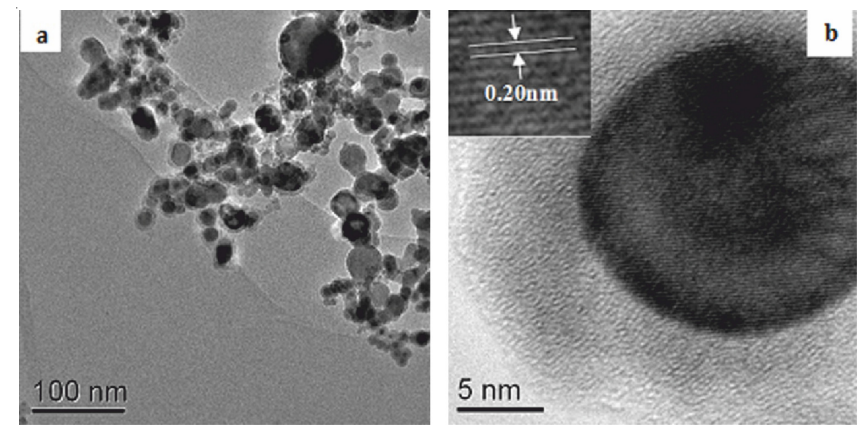

Fig. 1. (a) HRTEM micrograph and (b) HRTEM microscope microstructure of carbon encapsulated Ni nanoparticles

It is evident from Fig. 1(b) that the sample hold a clear core-shell structure, the core of the particles is $\mathrm{Ni}$ [dark globules in Fig. 1(b)] and the shell of the particles is carbons (light black shadow). The diameter of the core is about $18 \mathrm{~nm}$ and the thickness of the shell is about $8 \mathrm{~nm}$. It can be estimated from the inset image of Fig. 1(b) that the interlayer distance of crystalline Ni grain is about $0.20 \mathrm{~nm}$, corresponding to $\mathrm{Ni}$ (111) plane.

To further verify the crystalline structure of carbon-encapsulated Ni nanoparticles, XRD measurements were conducted. Fig. 2 shows the powder X-ray diffraction (XRD) pattern of carbon-encapsulated $\mathrm{Ni}$ nanoparticles. It can be seen from Fig. 2 that all the peaks in the curves are significantly broadened, corresponding to the nanocrystalline character of the particles, which is in agreement with the results of TEM characterizations. The broad peak at $2 \theta=26.24^{\circ}$ can be attributed to the diffractions of (002) planes of the hexagonal graphite structure, suggesting that the samples contain the significant amount of graphitic carbon, where the broad diffraction signal from $21-30^{\circ}$ is possible from amorphous carbon. Whereas other peaks positions appearing at $2 \theta=44.52^{\circ}, 51.88^{\circ}, 76.40^{\circ}$, $92.96^{\circ}$ and $98.48^{\circ}$ are well corresponding to the diffractions of (111), (200), (220), (311) and (222) planes of metallic $\mathrm{Ni}$, 


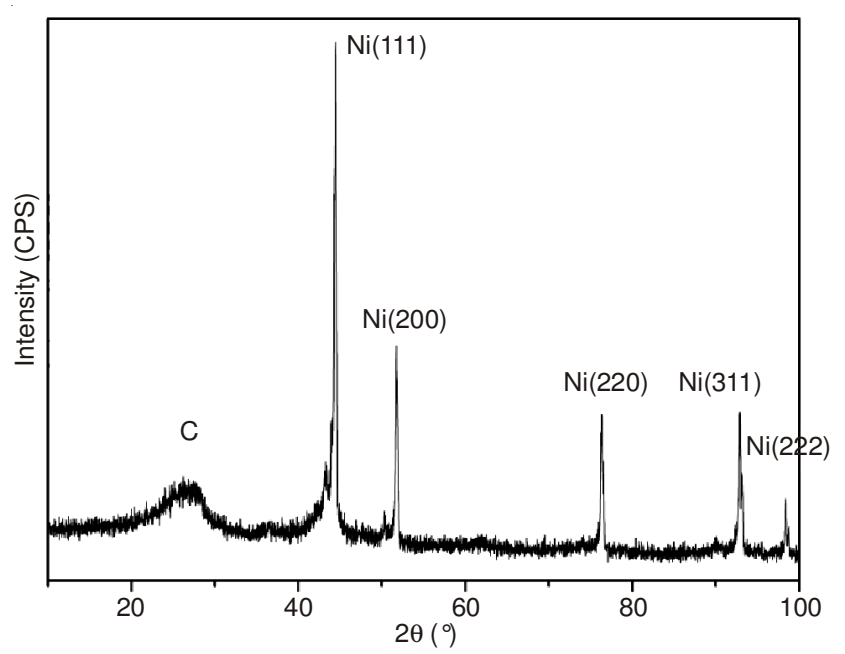

Fig. 2. XRD pattern of carbon encapsulated Ni nanoparticles

respectively. All these diffraction peaks can be perfectly indexed to the face centered cubic (FCC) crystalline structure of $\mathrm{Ni}$, which is in accordance with that of the standard spectrum (JCPDS No. 04-0850). The XRD pattern indicates the products of the present experiments consist of FCC Ni and amorphous carbon. In addition, no diffraction peaks assigned to metal oxides or Ni carbide compounds phase were detected.

The grain size of the metal core can be calculated from half widths of the major diffraction peak (111) according to Scherrer formula: $\mathrm{d}=\frac{0.89 \lambda}{\mathrm{B} \cos \theta}$, where $\mathrm{d}$ represents the grain size; $\lambda$ is the wavelength of the $\mathrm{X}$-ray $\left(\mathrm{CuK}_{\alpha}, 1.54056 \AA\right)$; $\theta$ is the diffraction angle of the peak; and $\mathrm{B}$ stands for the full width at half-height of the peaks (in radian) given by $\mathrm{B}^{2}=$ $\mathrm{Bm}^{2}-\mathrm{Bs}^{2}$, the effect of instrumental broadening on the reflection peaks was calibrated. The crystallite sizes of encapsulated $\mathrm{Ni}$ nanoparticles are $20 \mathrm{~nm}$ that was calculated from measured values for the spacing of the (111) plane. It can be seen that the mean particle size of the core determined by TEM is in good agreement with the average crystallite size calculated by Scherer formula from the typical XRD patterns. It suggests that the core of most $\mathrm{Ni}$ particles possible composed of one grain, i.e., Ni particles are single crystals.

The chemical compositions of carbon-encapsulated $\mathrm{Ni}$ nanopowders were examined by X-ray energy dispersive spectrometry (XEDS) (Fig. 3). It can be seen from Fig. 3 that the products are mainly composed of elements $\mathrm{Ni}$ and $\mathrm{C}$. The elements $\mathrm{Ni}$ characteristic peaks show that the core of the particles is $\mathrm{Ni}$ and the elements $\mathrm{C}$ peaks positions show that the shell of the particles is carbon. According to the XEDS analysis, except for elements $\mathrm{Ni}$ and $\mathrm{C}$, no oxygen element or other undesired byproducts were detected. X-ray energy dispersive spectrometry analysis proved that this technique has successfully overcome the problem of oxidation of the core metal nanoparticles.

Fig. 4 shows the magnetization hysteresis loop of the samples. A slight hysteresis in the magnetization curve indicates that either the particles are multidomain or that moments between individual particles are interacting. From Fig. 4, the saturation magnetization (Ms), the remanent magnetization

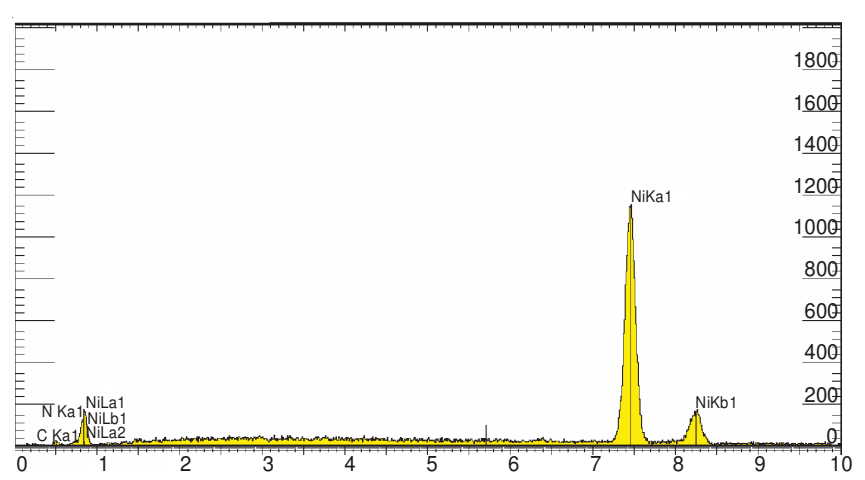

Fig. 3. XEDS of carbon encapsulated Ni nanoparticles

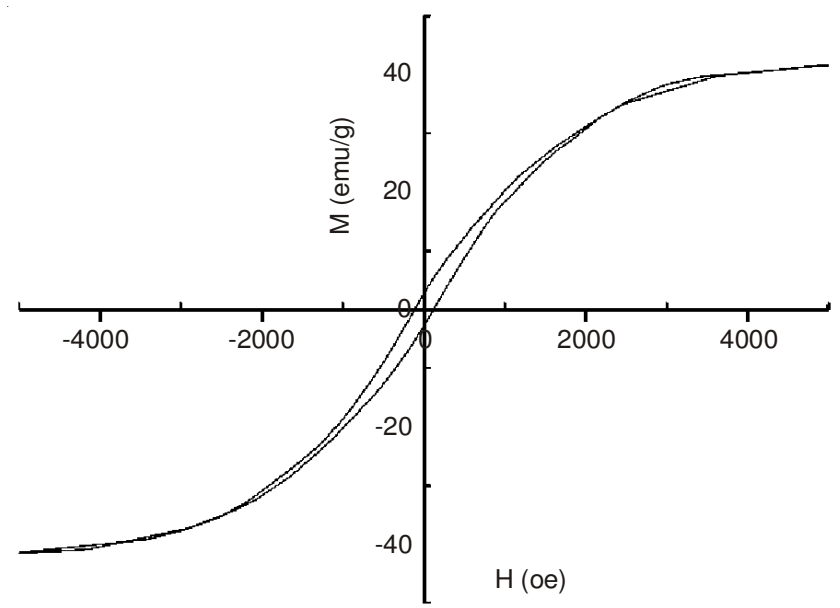

Fig. 4. Hysteresis loop of carbon encapsulated Ni nanoparticles

(Mr) and the coercivity (Hc) value of the particles are 42.3 emu/g, $2.62 \mathrm{emu} / \mathrm{g}$ and 103Oe, respectively. This result indicates the formed particles possess soft magnetic properties. The coercivity $(\mathrm{Hc})$ value of the particles is much higher than that of bulk $\mathrm{Ni}$ and the result is perhaps caused by many reasons, since the coercivity is sensitive to many structural parameters such as the internal stress, orientation, defects and shapes. In this study, the higher value of coercivity is probably due to their strong magnetic anisotropy and the ordered alignment of the nanoparticles in the carbon shell. The increased coercivity enables the material to bear a larger degree of demagnetization that is necessary for the application in the high-density magnetic recording materials. The saturation magnetization (Ms) of $\mathrm{Ni}$ seen from the $\mathrm{M}-\mathrm{H}$ plot is 42.3 emu/g, which is less than that of bulk $\mathrm{Ni}(\mathrm{Ms}=58 \mathrm{emu} / \mathrm{g})$ as reported. This can be attributed to the diamagnetic carbon of the carbon layers on the surface of the Ni nanoparticles and the randomness of surface spin and crystal direction of $\mathrm{Ni}$ nanocrystal. The properties of the magnetic material can be characterized using remanence ratio $(\mathrm{Mr} / \mathrm{Ms})$, the remanence ratio of the sample is $6.2 \%$ (Fig. 4), which indicate that the products show a paramagnetic property at room temperature.

\section{Conclusion}

- Carbon-encapsulated Ni nanoparticles with uniform size, well-dispersed and spherical shape were successfully prepared by DC arc plasma method, which own clear core-shell structure, the core of the particles is $\mathrm{Ni}$ and the shell of the particles is disorder carbons. 
- The crystalline structure of the encapsu-lated Ni nanoparticles is FCC structure. The particle size of the nanocapsules ranges from 15 to $40 \mathrm{~nm}$, with an averaged value about 30 $\mathrm{nm}$. The particle diameter of the core is about $18 \mathrm{~nm}$ and the thickness of the shells is about $6-8 \mathrm{~nm}$ and the magnetic properties are paramagnetic.

\section{ACKNOWLEDGEMENTS}

This work was supported by the National Natural Science Foundation of China (No. 51261015) and the Natural Science Foundation of Gansu Province, China (No. 1308RJZA238).

\section{REFERENCES}

1. S. Subramoney, Adv. Mater, 10, 1157 (1998).

2. M. Bystrzejewski, K. Pyrzynska, A. Huczko and H. Lange, Carbon, 47, 1201 (2009)

3. J. Dobson, Drug Dev. Res., 67, 55 (2006)

4. H. Tokoro, T. Nakabayashi, S. Fujii, H. Zhao and U.O. Häfeli, J. Magn. Magn. Mater, 321, 1676 (2009).

5. C. Baker, S.K. Hasanain and S.I. Shah, J. Appl. Phys., 96, 6657 (2004).

6. V.P. Dravid, J.J. Host, M. Teng, B.E.J. Hwang, D.L. Johnson, T.O. Mason and J.R. Weertman, Nature, 374, 602 (1995).

7. X.F. Zhang, X.L. Dong, H. Huang, B. Lv, X.G. Zhu, J.P. Lei, S. Ma, W Liu and Z.D. Zhang, Acta Mater, 55, 3727 (2007).
8. L.Z. Zhu, J.W. Ma, N.Q. Jia, Y. Zhao and H.B. Shen, Colloids Surf. B, 68, 1 (2009).

9. Z.P. Xu, Q.H. Zeng, G.Q. Lu and A.B. Yu, Chem. Eng. Sci., 61, 1027 (2006).

10. U. Narkiewicz, M. Podsiadly, W. Arabczyk, M.J. Wozniak and K.J. Kurzydlowski, Mater. Sci. Eng. C, 27, 1273 (2007).

11. G.X. Zhu, X.W. Wei and S. Jiang, J. Mater. Chem., 17, 2301 (2007).

12. D.S. Jacob, I. Genish, L. Klein and A. Gedanken, J. Phys. Chem. B, 110, 17711 (2006)

13. M. Bystrzejewski, A. Huczko, H. Lange, P. Baranowski, G. Cota-Sanchez, G. Soucy, J. Szczytko and A. Twardowski, Nanotechnology, 18, 145608 (2007).

14. W. Wu, Z. Zhu, Z. Liu, Y.N. Xie, J. Zhang and H.T. Hu, Carbon, 41, 317 (2003).

15. C.F. Wang, J.N. Wang and Z.M. Sheng, J. Phys. Chem. C, 111, 6303 (2007).

16. A.S. Gandhi, V. Jayaram and A.H. Chokshi, Mater. Sci. Eng. A, 304306, 785 (2001).

17. J.P. Huo, H.H. Song and X.H. Chen, Carbon, 42, 3177 (2004).

18. R.S. Ruoff, D.C. Lorents, B. Chan, R. Malhotra and S. Subramoney, Science, 259, 346 (1993).

19. Y. Saito, T. Yoshikawa, M. Okuda, N. Fujimoto, S. Yamamuro, K. Wakoh, K. Sumiyama, K. Suzuki, A. Kasuya and Y. Nishina, J. Appl. Phys., 75, 134 (1994)

20. Z.Q. Wei, T.D. Xia, L.F. Bai, J. Wang, Z.G. Wu and P.X. Yan, Mater. Lett., 60, 766 (2006). 